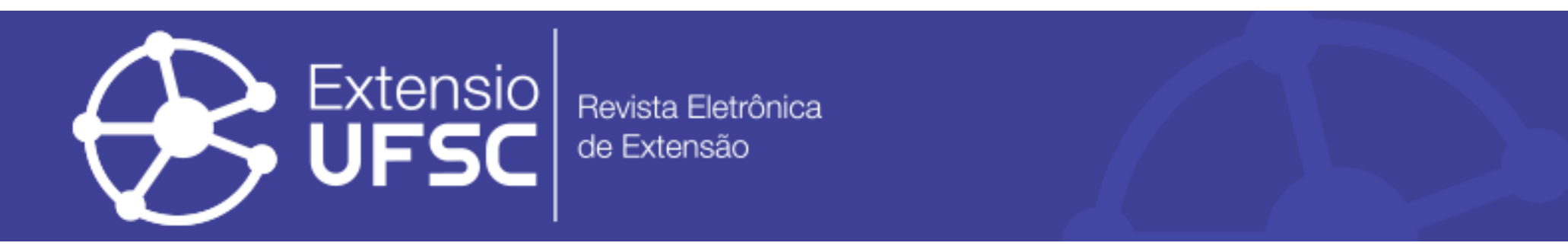

\title{
PROJETO RONDON: RELATOS DE UM PROFESSOR RONDONISTA DA UFSC
}

\author{
Edmilson Rampazzo Klen \\ Universidade Federal de Santa Catarina \\ edmilson.rk@ufsc.br
}

\begin{abstract}
Resumo
O Projeto Rondon, coordenado pelo Ministério da Defesa, pela sua história e vasta abrangência de atuação no país pode ser considerado como um projeto de extensão de grande impacto positivo no Brasil. Tem como principais objetivos contribuir para a formação do universitário como cidadão, integrar o universitário ao processo de desenvolvimento nacional, consolidar no universitário brasileiro no sentido de responsabilidade social e coletiva, em prol da cidadania, do desenvolvimento e da defesa dos interesses nacionais e estimular no universitário a produção de projetos coletivos locais, em parceria com as comunidades assistidas. Suas regiões prioritárias de atuação são aquelas com maiores índices de pobreza e exclusão social, bem como áreas isoladas do território nacional que necessitem de maior aporte de bens e serviços. O Projeto Rondon acontece duas vezes ao ano, sempre nos períodos de janeiro e julho, período de recesso escolar, reforçando a atividade voluntária de todos os participantes. A UFSC tem atuado intensamente no Projeto Rondon desde a sua criação. Este trabalho apresenta o envolvimento da UFSC e alguns relatos de experiência do autor que, de longa data, atua como rondonista e coordenador do Projeto Rondon na UFSC.
\end{abstract}

Palavras-chave: Projeto Rondon. Projeto de Extensão. Rondonistas. Voluntários.

\section{RONDON PROJECT: REPORTS OF A TECHAER}

\begin{abstract}
The Rondon Project, coordinated by the Brazilian Defense Ministry, has a long lasting in view of history and a vast scope of action in the country. It is considered an extension project with a great positive impact in Brazil, having as main missions: the contribution to the formation of undergraduate students as citizens; the integration of them in the national development process; the raising of awareness about the sense of social and collective responsibility in the favor of citizenship; the development and defense of national interests; and the motivation of the students to carry out local collective projects in partnership with assisted communities. The project's priority geographic areas of action are cities with high rates of poverty and social exclusion, as well as remote areas that need great input of goods and services. The Rondon Project takes place twice a year usually in January and July (University Summer and Winter breaks) reinforcing the volunteer approach of the initiative. The Federal University of Santa Catarina (UFSC) has been active in the Rondon Project since its beginning. This work presents the involvement of UFSC and some personal experiences of the author who has coordinated the UFSC team over 10 times along the years.
\end{abstract}

Keywords: Rondon Project. Extension Project. Students. Volunteers.

\section{PROYECTO RONDON: RELATOS DE UN PROFESOR}

\section{Resumen}

El proyecto Rondon, coordinado por el Ministerio de Defensa, en virtud de su historia y vasta amplitud de actuación en el país, puede ser considerado un proyecto de extensión de gran impacto positivo en Brasil. Sus principales objetivos son contribuir con el desarrollo de la formación ciudadana, la integración al proceso de desarrollo nacional y el sentido de responsabilidad social y colectiva de los estudiantes universitarios a través de la ejecución de proyectos en colaboración con las comunidades locales. Las áreas prioritariamente seleccionadas para su implantación son las que presentan mayores niveles de pobreza y exclusión social, así como las que se encuentran en zonas geográficamente aisladas y que, por esa razón, registran carencias en el suministro de bienes y servicios. El Proyecto Rondon se ejecuta dos veces por año, siempre durante los meses de enero y julio, coincidiendo con los períodos de receso escolar, de manera que se pueda incentivar la participación voluntaria del mayor número posible de participantes. Este trabajo presenta el involucramiento de la UFSC en esta actividad así como algunos relatos de experiencias del autor, quien viene desempeñándose desde mucho tiempo atrás como rondonista y coordenador del Proyecto Rondon en la UFSC.

Palavras clave: Proyecto Rondon. Proyecto de Extensión. Estudiantes. Voluntarios. 


\section{INTRODUCÃO}

O Projeto Rondon, coordenado pelo Ministério da Defesa, pela sua história e vasta abrangência de atuação no país pode ser considerado como um projeto de extensão de grande impacto positivo no Brasil. Tem como principais objetivos contribuir para a formação do universitário como cidadão, integrar o universitário ao processo de desenvolvimento nacional, consolidar no universitário brasileiro no sentido de responsabilidade social e coletiva, em prol da cidadania, do desenvolvimento e da defesa dos interesses nacionais e estimular no universitário a produção de projetos coletivos locais, em parceria com as comunidades assistidas.

Suas regiões prioritárias de atuação são aquelas com maiores índices de pobreza e exclusão social, bem como áreas isoladas do território nacional que necessitem de maior aporte de bens e serviços. O Projeto Rondon acontece duas vezes ao ano, sempre nos períodos de janeiro e julho, período de recesso escolar, reforçando a atividade voluntária de todos os participantes. A UFSC tem atuado intensamente no Projeto Rondon desde a sua criação.

A história do Projeto Rondon pode ser dividida em duas fases. Iniciado através de uma ideia durante a realização de um trabalho de sociologia em 1966 na Escola de Comando e Estado-Maior do Exército, teve a sua primeira operação em julho de 1967 quando 30 estudantes e dois professores deixaram o Rio de Janeiro com destino à Rondônia. A bordo de um avião disponibilizado pelo antigo Ministério do Interior, por 28 dias, o grupo realizou coleta de dados, pesquisas e atendimentos médicos na região. Assim como para este projeto, o nome do Estado de Rondônia foi dado em homenagem ao Marechal Cândido Rondon pelos importantes trabalhos de exploração, desenvolvimento e unificação nacional. Essa primeira fase, de cunho mais assistencialista, durou até 1989, estando a Universidade Federal de Santa Catarina - UFSC também presente em várias operações.

Toma-se como início da segunda fase a solicitação da União Nacional dos Estudantes - UNE, em 2015, com retomada do Projeto Rondon pelo Governo Federal, agora em um formato mais educativo, mas ainda visando o desenvolvimento comunitário local, bem como fortalecimento da cidadania. Da mesma forma que em sua primeira fase, a UFSC tem atuado com grande frequência.

O Projeto Rondon comemorou em 2015 o aniversário de 10 anos de seu relançamento, uma marca importante para esse que se apresenta como um dos principais programas de extensão universitária do Brasil. Nessa década de existência, a Universidade 
Federal de Santa Catarina foi uma Instituição de Ensino com relevante engajamento, até 2018 alcançando a participação em 28 operações realizadas desde 2005. Estas participações foram responsáveis por levar 224 alunos de diferentes cursos de graduação da UFSC para lugares, escolhidos pelo Ministério da Defesa, onde o conhecimento desses estudantes pudesse trazer benefícios à população local.

Apesar disso, o principal objetivo a que o projeto se propõe é o de transformar os rondonistas, nome dado aos professores e alunos que se voluntariam para participar deste projeto. Antes de 2005, quando a retomada do Rondon ainda era um projeto defendido pela União Nacional dos Estudantes (UNE), já se falava da importância do projeto para a criação de uma consciência de compromisso social em um grupo onde a maioria é de jovens prestes a entrar no mercado de trabalho.

A participação no Projeto Rondon é aberta a todas as Instituições de Ensino Superior do Brasil. Devem, primeiramente, fazer a sua inscrição no site oficial do projeto, aguarda-se abertura do Edital pelo Ministério da Defesa, que escolhe antecipadamente as regiões, costumeiramente recebendo um nome relacionado ao local precedido por “Operação", como exemplo da Figura 01.

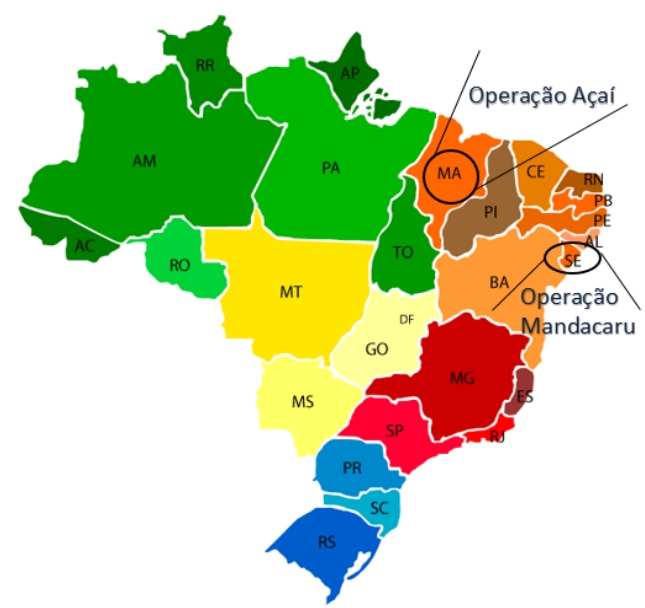

Fig. 1. Exemplo de regiões selecionadas pelo Ministério da Defesa para o Projeto Rondon

Baseado nas orientações de edital, a escrita de uma proposta de participação no Projeto Rondon, as Instituições de Ensino Superior (IES) devidamente inscritas, poderão enviar uma proposta de projeto de atividades para cada "Operação" disponibilizada. Estas propostas seguem a orientação de serem propostas A ou B, onde as atividades "A" englobam as áreas de cultura, direitos humanos e justiça, educação, saúde e a área "B" voltada para comunicação, meio ambiente, trabalho, tecnologia e produção. Nesta edição o 
grupo da UFSC vai desenvolver atividades voltadas para a área "A". As propostas devem ser enviadas de forma anônima, ou seja, não deve apresentar nome da IES, de professores e alunos para que o processo de avaliação seja igualitário a todas as IES, independente de sua região de atuação, número de cursos, entre outros.

No caso da aprovação da proposta, as responsabilidades da IES, bem como as do Ministério da Defesa, que coordena o Projeto Rondon, são estabelecidas no Termo de Cooperação, assinado pela autoridade maior da IES ou por representante por ela indicado e pelo Coordenador-Geral do Projeto Rondon.

\section{PARTICIPAÇÕES NO PROJETO RONDON PELA UFSC}

Desde o relançamento, em 2005, o Projeto Rondon realizou 76 operações, em 1.142 municípios de 24 unidades da federação, com a participação de 2.170 instituições de ensino superior e 21.436 rondonistas (universitários e professores), alcançando cerca de 2 milhões de pessoas (MINISTÉRIO DA DEFESA, 2018).

Cada cidade atendida recebe duas IES, uma com projeto A e outra com B, cada IES leva uma equipe de dois professores e oito alunos, estes preferencialmente com mais da metade do curso realizado, além do acompanhamento de um militar, que permanecerão no município por um período de duas semanas.

No período de 06 a 22 de julho de 2018 a UFSC estará na sua 28a. participação Projeto Rondon na cidade de NIOAQUE, dentro da Operação Pantanal, que abrange 12 cidades do estado do Mato Grosso do Sul. A equipe da UFSC estará desenvolvendo diversas atividades relacionadas ao projeto de atividades A em parceria com a universidade UniLasse do Estado do Rio de Janeiro com projeto de atividades B.

A tabela a seguir apresenta alguns dados de operações do Projeto Rondon das quais a UFSC foi selecionada desde 2005:

\begin{tabular}{|l|c|c|c|}
\hline Ano & Nome da Operação & Cidade & Estado \\
\hline $\mathbf{2 0 1 8}$ & Pantanal & Nioaque & MS \\
\hline $\mathbf{2 0 1 7}$ & Tocantins & Natividade & TO \\
\hline $\mathbf{2 0 1 6}$ & Forte dos Reis Magos & Pedro Velho & RN \\
\hline $\mathbf{2 0 1 5}$ & Mandacaru & Baturité & CE \\
$\mathbf{2 0 1 5}$ & Itacaiúnas & Araguatins & TO \\
\hline $\mathbf{2 0 1 4}$ & Velho Monge & Alto Longá & PI \\
$\mathbf{2 0 1 4}$ & Guararapes & Pedras de Fogo de Açucar & PB \\
\hline $\mathbf{2 0 1 3}$ & São Francisco & Maracanã & AL \\
$\mathbf{2 0 1 3}$ & Forte do Presépio & Olinda Nova do Maranhão & MA \\
\hline $\mathbf{2 0 1 2}$ & Pai Francisco & Ponta de Pedras & PA \\
\hline 2012 & Açaí & &
\end{tabular}




\begin{tabular}{|l|c|c|c|}
\hline $\mathbf{2 0 1 1}$ & Rio dos Siris & Ilha das Flores & SE \\
$\mathbf{2 0 1 1}$ & Seridó & Florânia & RN \\
$\mathbf{2 0 1 1}$ & Zabelê & Picos & PI \\
\hline $\mathbf{2 0 1 0}$ & Mamoré & Vale do Anari & RO \\
$\mathbf{2 0 1 0}$ & Catirina & Igarapé do Meio & MA \\
$\mathbf{2 0 1 0}$ & Centro-Nordeste & Ichu & BA \\
\hline $\mathbf{2 0 0 9}$ & Nordeste-Sul & Restinga Seca & RS \\
$\mathbf{2 0 0 9}$ & Centro-Norte & Rionte Alegre & PA \\
$\mathbf{2 0 0 9}$ & Centro-Norte da Eva & AM \\
\hline 2008 & Inverno 2008 & Tomar do Geru & SE \\
$\mathbf{2 0 0 8}$ & Xingu & Uruará & PA \\
$\mathbf{2 0 0 8}$ & Grão-Pará & São Caetano de Odivelas & PA \\
$\mathbf{2 0 0 8}$ & Grão-Pará & Vitória do Jari & PI \\
\hline $\mathbf{2 0 0 7}$ & Centenário da Comissão Rondon & João Lisboa & PA \\
\hline 2007 & Amazônia Oriental & São Gabriel da Cachoeira & AM \\
\hline $\mathbf{2 0 0 6}$ & Amazônia 2006 & São Gabriel da Cachoeira & AM \\
\hline $\mathbf{2 0 0 5}$ & Amazonas 2005 & & \\
\hline
\end{tabular}

Além das operações apresentadas, ressalta-se que no período de 23 a 25 de setembro de 2015, a Universidade Federal de Santa Catarina, foi palco do II Congresso Nacional do Projeto Rondon (II CONGRESSO NACIONAL DO PROJETO RONDON, 2018) onde participaram cerca de 300 rondonistas de diversas partes do Brasil. Os trabalhos foram apresentados nas formas de pôsteres e/ou apresentações orais, contando um pouco das valiosas participações dos alunos e das diferentes instituições de ensino superior no Projeto Rondon oportunizando debates que realçaram o passado, o presente e projeções para o futuro do Projeto Rondon.

A UFSC tem atuado intensamente no Projeto Rondon, onde expressa-se aqui, a relevância do apoio da Pró-Reitoria de Extensão para o sucesso de suas atuações (PROEX, 2018).

\section{RESPONSABILIDADES}

A cada nova edição de edital do Projeto Rondon, segue-se uma série de trabalhos para que a UFSC possa participar, entre estas atividades, destaca-se as principais atividades e responsabilidades tanto da IES, dos professores como dos estudantes.

\section{1- Atividades e responsabilidades da IES}

Baseado nas orientações do Ministério da Defesa (PROJETO RONDON, 2018), são responsabilidades da Instituição de Ensino Superior - IES:

- observar o contido na Orientação às IES, na área de downloads; 
- designar, para cada uma de suas equipes, um professor-coordenador, com perfil para atividades de extensão universitária, para gerenciamento de grupos de trabalho e com excelente capacidade de relacionamento;

- designar o professor que fará a viagem precursora, lembrando que este deverá fazer parte da equipe que atuará no município;

- remeter à Coordenação-Geral do Projeto Rondon, após a viagem precursora, o quadro de atividades ajustado entre as duas equipes e o município;

- selecionar os rondonistas, organizar e preparar a(s) equipe(s) que participará(ão) da operação, de modo a cumprir o previsto no(s) plano(s) de trabalho;

- cadastrar seus rondonistas que participarão da operação;

- transportar a(s) equipe(s) entre o(s) local(is) de origem e a cidade de partida;

- desenvolver, no município designado, as ações previstas na proposta de trabalho;

- dar conhecimento e fazer cumprir, por seus rondonistas, o prescrito no Guia do Rondonista, que se encontra na área de download, e os prazos estabelecidos para as atividades da operação;

- apresentar o relatório de atividades no encerramento da operação;

- assinar e remeter à Coordenação-Geral do Projeto Rondon o Acordo de Cooperação da IES com o MD;

- remeter à Coordenação-Geral do Projeto Rondon o Termo de Compromisso do Rondonista e o Termo de Cessão de Uso de Imagem, assinado por cada rondonista (professores e alunos);

- alertar os integrantes das equipes sobre a obrigatoriedade de participação em todos os eventos da operação;

- obedecer aos prazos estabelecidos neste convite; e

- exigir das equipes o cumprimento do contido no Guia dos Rondonistas

\section{2- Atividades e responsabilidades do professor}

- Leitura detalhada do novo edital;

- Preparação e divulgação da chamada de seleção de professores e de alunos (reuniões, palestras, desenvolvimento e aplicação de processo de seleção);

- Escrita, junto ao(s) grupo(s) selecionado(s), escrita do(s) projeto(s) (planejamento de oficinas e palestras para a região selecionada);

- Acompanhamento do processo de avaliação do Ministério da Defesa e, quando necessário, aplicação de recursos; 
- Aprimoramento de testes das oficinas selecionadas com grupo da UFSC (reuniões, geração de procedimentos e planejamento das realizações, etc.);

- Viagem precursora, que é a ida, obrigatória, do coordenador do grupo, ao local selecionado para planejamento da Operação na cidade selecionada (conhecimento, seleção e preparação da infraestrutura local para a realização das atividades dos rondonistas).

- Detalhamento das oficinas selecionadas com grupo da UFSC (reuniões, geração de procedimentos e planejamento das realizações, etc.);

- Preparação da viagem do grupo da UFSC (logística, materiais, etc.);

- Realização da Operação do Projeto Rondon (apresentada no sistema Sigpex);

- Realização do fechamento da atuação do grupo da UFSC na devida Operação (relatórios)

- Atenção aos prazos de novo edital

Exemplifica-se aqui ainda, algumas atividades, não costumeiras, mas que foram solicitados para os representantes do Projeto Rondon da UFSC:

- Auxílio no planejamento, preparação e participação no encontro de grupo de professores rondonistas com o Ministro da Defesa, na época, Sr. Celso Amorim, para solicitação de mais verbas para o Projeto Rondon (Figura 2).

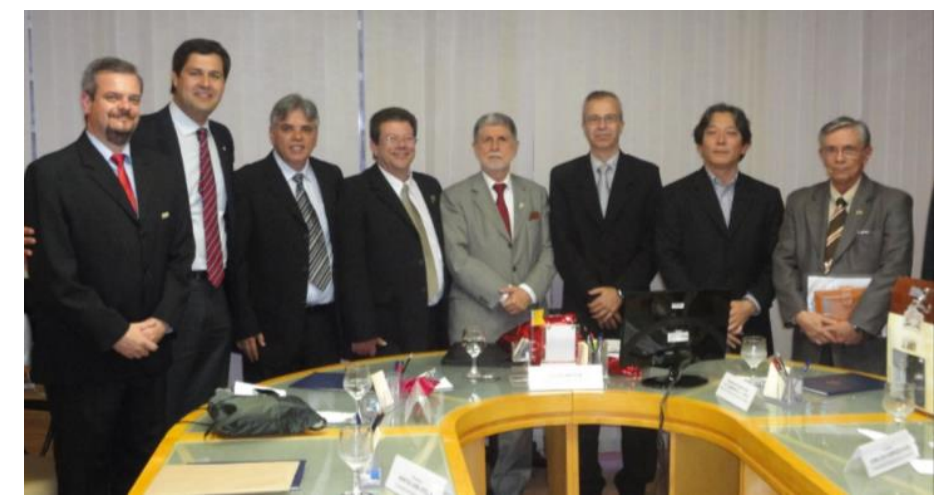

Fig. 2. Encontro com o Ministro da Defesa Sr. Celso Amorim, 2011

- Participação da posse do novo Comandante Geral do Projeto Rondon, ViceAlmirante fuzileiro naval Paulo Martino Zuccaro (Figura 3). 


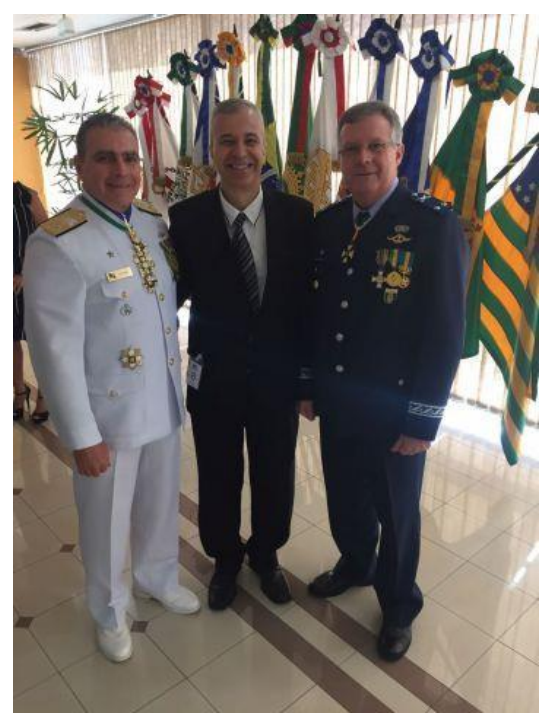

Fig. 3. Posse do Cmte Geral do Projeto Rondon, Vice-Almirante Paulo M. Zuccaro,2017

- Participação das reuniões bianuais de professores Rondonistas.

- Coordenação do II Congresso Nacional do Projeto Rondon, na UFSC, 2015 (Figura 4).

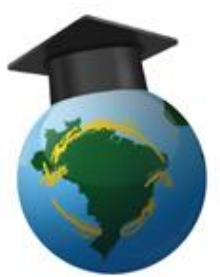

II Congresso Nacional do

PROJETO RONDON

Fig. 4. II Congresso Nacional do Projeto Rondon realizado nas dependências da UFSC

- Contato com a coordenação do curso de Medicina da UFSC onde se solicitou a liberação de alunos que estejam no internato para participarem do Projeto Rondon.

- Participação de outros eventos do Rondon respaldados pelo Ministério da Defesa.

\section{3- Atividades e responsabilidades dos estudantes rondonistas}

São compromissos de todo rondonista antes da operação:

- Estudar a região para onde a IES atuará;

- Preparar atividades de acordo com as necessidades observadas no estudo da região;

- Objetivar a criação de atividades que possam se tornar multiplicadoras;

- Participar das reuniões preparatórias do grupo;

- Treinar suas atividades e participar dos treinos de outros rondonistas;

- Tomar conhecimento das diretrizes, direitos e deveres do rondonista;

- Assinar o "Termo de Compromisso do Rondonista" e o "Termo de Cessão de Uso de Imagem" 
São compromissos de todo rondonista durante a operação:

- Portar sempre o seu documento de identidade original e o crachá de rondonista.

- Usar camiseta de rondonista durante todas as atividades da operação.

- Cumprir os horários estabelecidos, evitando transtornos para os demais membros da equipe e para a execução das atividades.

- Zelar pelo material que lhe for confiado.

- Ser solidário com os integrantes da equipe.

- Respeitar os costumes, hábitos, tradições e crenças da comunidade onde estiver.

- Não fazer promessas à comunidade em nome do Projeto Rondon, nem em nome de sua IES.

- Não se envolver em questões políticas ou religiosas.

- Ser discreto, não divulgar fatos e informações locais indiscriminadamente e tampouco criticar ou desacreditar abertamente as autoridades locais.

- Não aceitar qualquer tipo de pagamento ou vantagem pessoal como retribuição da comunidade por um trabalho realizado.

- Manter as discussões particulares ou de ordem técnica nos limites de sua equipe ou grupo de trabalho.

- Fazer prevalecer o interesse coletivo sobre o individual.

- Não estabelecer contatos ou prestar declarações em nome do Projeto Rondon. Quando der entrevistas, falar em seu próprio nome.

\section{SELEÇÃO DOS ALUNOS}

O Ministério da Defesa dá liberdade para que cada IES tenha seu próprio método de seleção de estudantes para a formação do grupo que a representará.

Na UFSC, geralmente, faz-se primeiramente, uma chamada pelo site da PROEX assim como em outros canais de comunicação internos como, por exemplo, a Agência de Comunicação (Agecom) estipulando local e horário para uma reunião de seleção.

Para esta seleção observa-se os seguintes critérios para a candidatura:

- Ser aluno de graduação da UFSC e estar cursando a segunda metade do curso, com preferência para os últimos semestres; 
- Ter ótimo currículo (histórico, Índice de Aproveitamento Acumulado - IAA, estágios, monitorias etc.);

- Ter disponibilidade de horário para elaborar e realizar o projeto.

- Fazer inscrição on-line (pelo site da PROEX)

- Participar da reunião de seleção, presença obrigatória para estudantes do Campus Trindade (Florianópolis). Estudantes de outros campi (Araranguá, Blumenau, Curitibanos e Joinville) a presença não é obrigatória, porém deverão realizar a inscrição e enviar o trabalho escrito por e-mail (projeto.rondon@contato.ufsc.br), até o horário da referida reunião seletiva.

- $\mathrm{Na}$ reunião de seleção, os candidatos deverão entregar, trabalho escrito, contextualização sobre o território onde será desenvolvido à operação (de 500 a 800 palavras) assim como duas ideias de atividades abrangidas dentro das áreas temáticas determinadas pela UFSC para esta operação (projetos A ou B).

Somente depois da formação do grupo é que se estabelecerão reuniões e devidos prazos para a geração da proposta do projeto de atividades

$\mathrm{Na}$ seleção dos alunos, não há restrição para o curso, mas busca-se alunos com bom IAA, com mais da metade do curso realizado e que se dispõem a um trabalho social e colaborativo, não se esquecendo também, da disposição por aventura. Integrar conhecimentos é outra das expectativas de quem participa do Rondon ainda que não sejam diretamente relacionadas ao seu curso. A interdisciplinaridade dentro das atividades ajuda a construir relações entre o que aprendem na graduação e o resto do mundo. Essa convergência de conhecimento também tem relação com o contato com colegas de outros cursos de graduação, algo que ajuda a observar os vínculos entre ciências diferentes.

\section{Visão de participação dos estudantes rondonistas}

Baseado em um longo período de atuação no Projeto Rondon pela UFSC, onde foi oportuno estar participando pela $12^{\mathrm{a}}$. vez, na maioria dos casos como professor coordenador, é possível ter uma visão, de uma forma geral, do resultado da participação dos estudantes ressaltando a importância desta extensão como fator potencializador do ensino curricular e própria preparação do estudante pós universidade.

Mesmo antes da atuação como rondonistas, muitos estudantes já visualizam a perspectiva de encontrar uma realidade diferente da sua conhecida, e com isso pensar em como ajudar a melhorá-la, como um fator essencial na decisão de tentar participar do projeto. Por várias vezes, pode-se sentir o sentimento de que "por mais complicado que seja o cenário que venhamos a encontrar é difícil imaginar que se possa mudar a realidade 
dessas pessoas em apenas duas semanas de atuação do Rondon, mas a sua participação nesta tentativa de melhorá-lo será levada para sempre."

Algumas atividades realizadas em operações do Rondon puderam ser devidamente mensuradas, como por exemplo, o caso aplicação da oficina "Banheiro Seco" em um Colégio Agrícola na cidade de Natividade-TO dentro da operação Tocantins em janeiro de 2017. Esta atividade foi considerada de grande multiplicidade pois sua implantação foi voltada para os professores do colégio e passou a fazer parte da disciplina "Construções Rurais" com diversas considerações da difusão desta tecnologia com uso de matérias primas de baixo custo disponíveis na região. Esta atividade, além de receber grande repercussão dos meios de comunicação locais durante a sua realização, foi premiada no III Congresso Nacional do Projeto Rondon (III CONGRESSO NACIONAL DO PROJETO RONDON, 2018), realizado em Brasília-DF em 2017. (Figura 5)

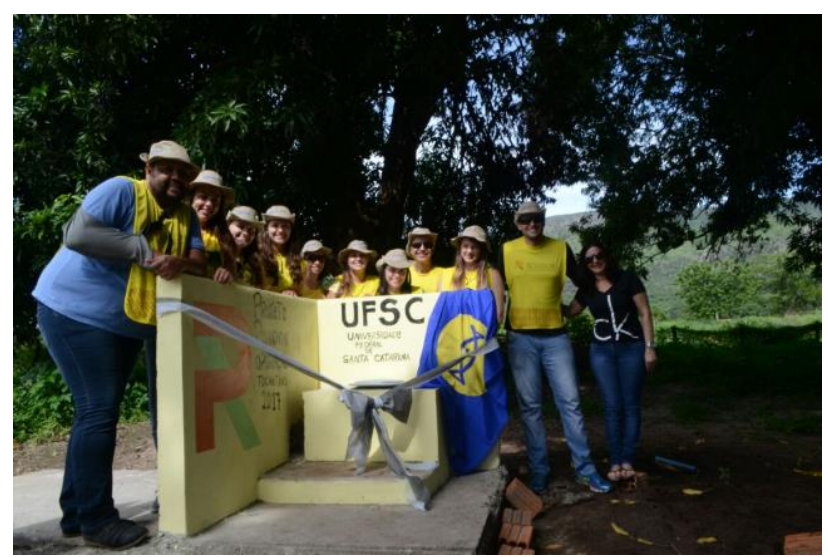

Fig. 5. Rondonistas da UFSC na entrega do Banheiro Seco no Colégio Agropecuário em Natividade-TO

$\mathrm{Na}$ maioria das vezes, o sucesso da atuação dos rondonistas não tem a oportunidade de ser medido como o caso anterior mas se refletem de outra forma. Como exemplo, dentro da operação Forte dos Reis Magos realizada em julho de 2016 no Estado do Rio Grande do Norte, após um dia de atuação ainda na primeira semana de trabalhos na comunidade de Cuité, na cidade de Pedro Velho-RN, a população local, como forma de mostrar sua gratidão, se reuniu e ofereceu um almoço com comidas típicas para os rondonistas na segunda semana do projeto. Ainda nesta operação, a prefeitura local realizou uma cerimônia de despedida com homenagens a todos os rondonistas, onde foi ressaltado a abrangência das comunidades e população atendidas além da cordialidade e atenção dispensadas pelos rondonistas.

A satisfação dos habitantes do município pela oportunidade de receber orientações sobre diferentes temas das atividades realizadas foi um dos fatores que mais chamou a atenção e emocionou os rondonistas. Para os voluntários, mais do que ensinar técnicas, "o 
importante é conhecer uma cultura diferente e ver, de perto, as angústias e as alegrias de uma população humilde".

Isto indica que estas ações proporcionam o desenvolvimento das comunidades locais através de técnicas aprendidas na universidade, como demonstra também que, este contato e troca experiências, possibilita aos rondonistas um melhor desenvolvimento de sua cidadania e valorização da diversidade cultural do nosso país.

\section{CONCLUSÃO}

A experiência adquirida pelos estudantes em todo o processo de participação do Projeto Rondon, desde um concorrido processo de seleção, passando pela preparação da proposta, até a operação propriamente dita, é uma valiosa e indescritível oportunidade que fica para a vida toda.

São inúmeros os exemplos de estudantes que mudaram a forma de ver o mundo, quando do contato com comunidades carentes pelos interiores do Brasil, principalmente nas regiões Norte e Nordeste. Alguns chegam a estudar, trabalhar e morar nesses locais depois de terminado o seu curso de graduação. Muitos se tornam profissionais engajados na visão do desenvolvimento do Brasil como um todo, em sua área de atuação.

Em adição, esta rica troca de experiência com as comunidades atendidas possibilita aos estudantes um melhor desenvolvendo de sua cidadania além de uma valorização da diversidade cultural de nosso País. Conhecer melhor a realidade brasileira, proporcionar o trabalho coletivo e a aplicação dos conhecimentos acadêmicos para o bem-estar de comunidades carentes são alguns dos benefícios do Projeto Rondon.

Como professor rondonista da UFSC, resumo o Projeto Rondon como um gesto de carinho pois, os estudantes rondonistas, se propõe, durante as suas férias, levar conhecimentos adquiridos no seu curso - ou mesmo da sua experiência de vida - a diferentes comunidades em qualquer lugar do país, com o intuito de melhorar a qualidade de vida de pessoas que nem conhece.

Este ato voluntário, ratifica importância desta extensão como fator potencializador do ensino curricular e do desenvolvendo de sua cidadania.

Caso haja interesse, os estudantes devem ficar atentos aos processos seletivos geralmente disponibilizados no site. Outras informações mais detalhadas desta e de outras Operações podem também ser acessadas pelo site oficial do Projeto Rondon (PROJETO RONDON, 2018). 


\section{REFERÊNCIAS}

MINISTÉRIO DA DEFESA, Projeto Rondon, Disponível em: $<$ https://www.defesa.gov.br/index.php/programas-sociais/projeto-rondon>, Acesso em: 27 maio 2018.

II CONGRESSO NACIONAL DO PROJETO RONDON, O Congresso, Disponível em: <http://congressorondon2015.ufsc.br/>, Acesso em: 02 junho 2018.

III CONGRESSO NACIONAL DO PROJETO RONDON, O Congresso, Disponível em: <http://www.congressorondon2017.unb.br>, Acesso em: 02 junho 2018.

PROEX, Pró-Reitoria de Extensão da Universidade Federal de Santa Catarina, Brasil, Disponível em: < http://proex.ufsc.br/projeto-rondon/>, Acesso em: 04 junho 2018.

PROJETO RONDON, Site oficial, Disponível em: $<$ https://projetorondon.defesa.gov.br/portal/operacao/pagina/id/40805/area/P/module /default>, Acesso em: 04 junho 2018.

Recebido em: 08/06/2018

Aceito em: 06/07/2018 